\title{
Yoga-An Alternative Form of Therapy in Patients with Blunt Chest Trauma: A Randomized Controlled Trial
}

\author{
Mallikarjun Gunjiganvi ${ }^{1} \cdot$ Purva Mathur $^{2} \cdot$ Minu Kumari $^{3} \cdot \operatorname{Karan}_{\text {Madan }^{4}} \cdot$ Atin Kumar $^{5}$ •

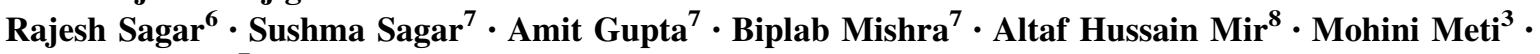 \\ Subodh Kumar ${ }^{7}$
}

\begin{abstract}
Background Yoga as alternative form of therapy has shown positive impact on pulmonary functions, exercise capacity, behavioral changes, and inflammation in non-trauma patients. However, the efficacy of Yoga has not been studied in chest trauma patients.

Methods This randomized controlled trial was conducted at level-1 Trauma Centre. Isolated chest injury patients were randomized into either standard physiotherapy or Yogatherapy groups. Patients in physiotherapy group received conventional chest physiotherapy and Yogatherapy group received a set of Yogic exercises in addition to conventional chest physiotherapy. Primary outcome measure was changes in pulmonary function tests (PFT) at 4 weeks of discharge. Secondary outcomes were changes in quality of life $(\mathrm{QoL})$, respiratory muscle strength and endurance, chest wall mobility, and levels of cytokines at 4 weeks. Data were analyzed using STATA v14.0.

Results A total of 89 eligible patients were randomized to physiotherapy $(n=46)$ and Yoga therapy $(n=43)$ groups. Demographic characteristics were comparable in both the groups. There were statistically significant improvements in PFT in the Yogatherapy group compared with physiotherapy with an increase in Forced vital capacity $(p=0.02)$ and Forced expiratory volume $(p=0.01)$ at 4 weeks. In addition, there were significant improvement in physical component of QoL, respiratory muscle endurance $(p=0.003)$ and axillary cirtometry $(p=0.009)$ in the Yogatherapy group. However, no statistically significant difference in the trends of cytokine markers seen between the groups. Conclusion Yoga was found to be effective in improving pulmonary functions and QoL in patients with chest trauma. (Trial registered at ctri.nic.in/clinicaltrials/login.php, numberREF/2016/05/011,287).
\end{abstract}

Subodh Kumar

subodh6@gmail.com

1 Sanjay Gandhi Post Graduate Institute of Medical Sciences, Specialty of Trauma Surgery, Apex Trauma Center, Lucknow, India

2 Department of Laboratory Medicine, JPN Apex Trauma Center, All India Institute of Medical Sciences, New Delhi, India

3 Division of Trauma Surgery and Critical Care, JPN Apex Trauma Center, All India Institute of Medical Sciences, New Delhi, India

4 Department of Pulmonary Medicine and Sleep Disorders, All India Institute of Medical Sciences, New Delhi, India
5 Department of Radiology, JPN Apex Trauma Center, All India Institute of Medical Sciences, New Delhi, India

6 Department of Psychiatry, All India Institute of Medical Sciences, New Delhi, India

7 Division of Trauma Surgery and Critical Care, JPN Apex Trauma Center, All India Institute of Medical Sciences, New Delhi, India

8 Department of Physiotherapy, JPN Apex Trauma Center, All India Institute of Medical Sciences, New Delhi, India 


\section{Introduction}

Trauma is the major cause of hospitalization worldwide $[1,2]$. It is also associated with high mortality and longterm disabilities [3, 4]. Chest injuries account for approximately $10-15 \%$ of total trauma burden with mortality ranging from 5 to $77 \%$ [5-7]. Chest wall, pleural and lung parenchymal injuries result in pain, impaired ventilation, impaired oxygenation and perfusion [8-10], and activation of the inflammatory cascade [11-13]. This altered inflammatory response results in systemic inflammation, acute lung injury, acute respiratory distress syndrome and multiorgan dysfunction syndrome [14, 15].

Pain, restricted chest wall movements, impaired oxygenation, higher grades of injuries lead to development of pulmonary complications. This combined with altered inflammatory response result in higher complication rates. Many observational studies analyzing higher grades of Thoracic Trauma Severity Score have demonstrated disastrous outcomes [14, 16-20]. Apart from physical dysfunction, psychological dysfunction is also significant among trauma survivors and affects quality of life in both short and long terms [21-26].

Implementation of early, effective chest rehabilitation with adequate pain control, aggressive chest physiotherapy modulates pulmonary dynamics (pain, chest wall movement, oxygenation, pulmonary functions). This minimizes pulmonary secretion retention, maximizes oxygenation, reexpands atelectatic lung and improves pulmonary functions; reduces infection and mortality rates; intensive care unit and hospital stay [27-29].

The holistic approach of Yoga involving deep breathing Pranayamas (breath control exercises) and Yogic asanas (Yoga postures) is well-established in non-trauma setting [30-32]. These Yogic practices have shown beneficial effects on cardio-pulmonary dynamics and performance, pulmonary functions, exercise capacity, immune system, mental health problems and quality of life (QoL) [33-44].

There is a dearth of literature about the effects of Yoga in trauma setting. Hence, this study was planned to examine the effect of Yoga in the clinical recovery and post injury outcomes of patients with blunt chest trauma.

\section{Materials and methods}

This prospective open-label randomized controlled study was carried out in the Division of Trauma Surgery and Critical Care at a Level I trauma center from June 2016 through August 2018. Study was approved by Institutional Ethics Committee and was registered with Clinical Trials
Registry-India (Trial registered at ctri.nic.in/clinicaltrials/ login.php, numberREF/2016/05/011,287).

\section{Study population}

Patients aged 18-65 years with isolated blunt chest trauma and who were managed non-operatively with or without thoracostomy tubes were recruited.

\section{Exclusion criteria}

Patients with injury duration $>24$ hours, requiring ventilator support, co-existing conditions like malignancies, coagulopathy, chronic systemic diseases, patients on hormone therapy, preexisting disability interfering with $\mathrm{Yo}$ gatherapy and pregnant women were excluded.

\section{Randomization}

Randomization was performed using computer-generated list of random numbers and allocation concealment was strictly followed. Patients were randomly allocated into standard chest physiotherapy (CTP) Group or Yogatherapy (YTP) Group. Provision for post-randomization exclusion was considered before start of study in the following instances: randomized patients requiring ventilator support for whom YTP could not be feasible and patients who wished to exit from the study due to multiple blood samplings required for the cytokines assessment. These post randomization exclusion criteria were set based on our previous experiences with trauma patients.

\section{Intervention}

All patients visiting emergency department with torso injuries were managed according to Advanced Trauma Life Support protocols. Relevant baseline investigations were done including arterial blood gas analyses, chest X-ray, pelvic X-ray and computed tomography of torso. After further screening for isolated blunt chest injuries, eligible patients were recruited. We have specific institutional protocols for analgesia and thoracostomy tube management in chest injury patients.

\section{Oxygenation strategy}

All the patients were administered supplemental oxygen by facemask during the primary survey and it was continued depending on requirement. 


\section{Analgesia protocol}

Institute has departmental standardized analgesia protocol in place for the management of chest trauma patients. The analgesia protocol used in patients with chest injuries was "Sandwich Analgesic Protocol therapy." Aim was to ensure that patients were given analgesics every sixhourly-paracetamol $(1000 \mathrm{mg})$ alternating with diclofenac $(50 \mathrm{mg})$. It was supplemented by regional Transdermal Drug Delivery System (Nu Patch ${ }^{\mathrm{TM}}$ manufactured by Zydus Cadila Limited-containing Diclofenac, Linseed oil, Methyl salicylate and menthol) on need basis. Medications were initially administered parenterally followed by orally as tolerated. Epidural analgesia was considered in patients with bilateral chest tubes, bilateral rib fractures, flail chest and those not responded to sandwich analgesia therapy. Opioids were not used in any patient. Same protocol was used both the groups.

\section{CTP group}

Patients received standard chest physiotherapy which included percussion, vibration, cough stimulation techniques, breathing exercises and mobilization from admission through discharge. At discharge, a set of instructions were given for home-based physiotherapy.

\section{YTP group}

Patients received Yogatherapy along with standard chest physiotherapy. Yogatherapy was delivered by trained Yoga instructor under supervision of medical experts for duration of upto maximum $1 \mathrm{~h}$ as tolerated on daily basis from admission through discharge. Patients were taught Pranayamas and then gradually moved on to Asanas. Patients were advised to continue Yoga at home after discharge and were given a printed Yoga booklet for reference in regional language with graphical illustrations. All patients in both arms were followed up at 1st, 2nd and 4th week of discharge.

\section{Outcome measures and data collection}

Primary outcome: Pulmonary functions (PFTs) at 4 weeks from discharge. Secondary outcomes: (1) QoL, Respiratory muscle endurance (RME), Respiratory muscle strength (RMS), Chest wall mobility (CWM) and Cytokine levels at 4 weeks from discharge; (2) Change in PFTs, RME and CWM over time from admission to 4 weeks follow-up; (3) Changes in Cytokines levels before and after intervention during hospital stay.
PFTs measured were Tidal Volume (VT), Forced Vital Capacity (FVC), and Forced Expiratory Volume in one Second (FEV1), Peak Expiratory Force (PEF) and FVC/ FEV1 ratio. RME was assessed by measuring Maximum voluntary ventilation (MVV). All values were recorded in liters using Spirolyser Q13 USB and Qflow ${ }^{\circledR}$ sensor (manufacturer: FIM medical, France) [45-48].

For QoL, World Health Organization (WHO) BREF 26, a standardized assessment instrument comprising of 26 items/questions was used. It has four main domains: physical, psychological, social relationships and environment domains [49].

RMS was assessed by diaphragm ultrasound for diaphragm movements and thickness. These variables were recorded both during resting and deep respiration using Siemens ACUSON S2000 and/or SonoSite M-Turbo [50].

In CWM, chest wall excursion during deep inspiration was measured at two sites (values expressed in centimeters) using measuring tape: at the level of nipple-Axillary cirtometry and xiphi-sternum-Thoracic cirtometry [51].

Serum cytokines Interleukin (IL)-2, IL-4, IL-8, IL-10, IL-12, Tumor necrosis factor (TNF)- $\alpha$ and Interferon (IFN)- $\gamma$ were measured. Blood samples were collected on day 0 , one hour before and $2 \mathrm{~h}$ after interventions on day 1 , $2,3,4$ or on the day of discharge; and one blood sample during follow-up at 4 weeks. Blood samples were centrifuged, stored at $-80{ }^{\circ} \mathrm{C}$ and analyzed at the end of study by enzyme linked immunosorbent assay technology using PowerWave $^{\mathrm{TM}}$ XS Microplate Reader by BioTek Instruments, Inc.

\section{Statistical analysis}

Sample size was calculated taking into consideration that Yoga would reduce the hospital stay by 1 day in patients with isolated chest trauma. Thus, initial sample size was 66 patients with $\beta$-power of $80 \%$ and $\alpha$-power of $5 \%$. However, patients were continuously recruited further even after completion of sample size. Data was collected in Microsoft Excel 2010 for windows. Statistical analysis was carried out using Stata v: 14.0 for windows. Data analysis was done as per protocol basis and presented in mean (SD), and frequency $(\%)$ and median and interquartile range (IQR) wherever required. Categorical variables were compared by chi-squared test and Fischer's test. Continuous variables were compared using independent-t test (for normal distribution) and Wilcoxon rank-sum (for nonnormal distribution). Overall variables between the groups over the time were compared by GEE (generalized estimating equation). 


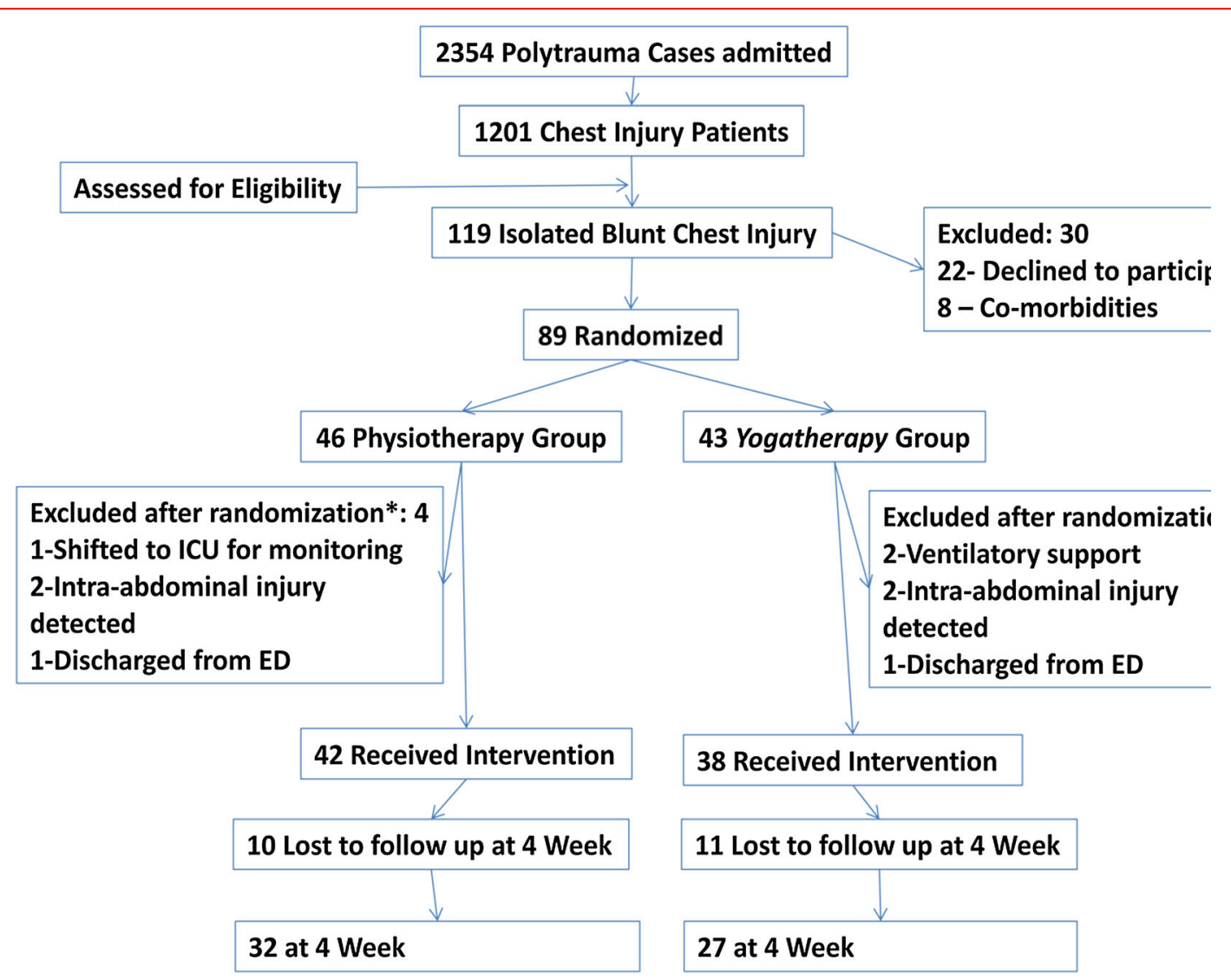

Fig. 1 Consort flowchart

\section{Results}

A total of 2354 poly-trauma patients were admitted during the study period. Out of which 119 patients with isolated blunt chest trauma were identified and 89 patients were recruited into the study. Post-randomization, nine patients were excluded from analysis for various reasons (Fig. 1).

Data from 80 patients, 42 in CTP group and 38 in YTP group were analyzed. All baseline demographic and clinical characteristics of both groups were comparable except the clinically detected pneumothorax which was significantly more in YTP group (Table 1).

Baseline outcome parameters viz. PFTs, RME, RMS, CWM, QoL, cytokines at admission were comparable in both groups except the physical component of QoL which was significantly low in Yogatherapy group $(p=0.05)$ (Table 2).

Supplemental oxygen was used in all patients in the primary survey and further on need basis. No patient received noninvasive assisted ventilation. Epidural Analgesia was used in 14 patients of CPT group and 17 patients of YTP group.

\section{Length of stay (LoS)}

The length of stay was comparable with mean $( \pm \mathrm{SD})$ $3.7 \pm 1.01$ days in CTP group and $4.3 \pm 2.03$ days in YTP group and was not statistically significant $(p=0.07)$. The chest tube duration was 3 (2.8-4) (median-IQR) days in physiotherapy group and 4 (3-5.8) days in YTP group with $\mathrm{p}$ of 0.05 indicating that chest tube was required for longer duration in YTP group. YTP group had statistically significant more pneumothorax $(p<0.001)$ and non-statistically significant hemothorax $(p=0.07)$ compared to CTP group at admission.

\section{Primary outcome measures}

There was statistically significant improvement in pulmonary functions at 4 weeks from discharge in YTP group with improvement in VT $(p=0.04, \mathrm{FVC}(p=0.02)$, FEV1 $(p=0.01)$, PEF $(p=0.002)$ of the YTP group. (Table 3$)$.

\section{Secondary Outcome Measures}

There was no statistical difference in all four domains of the QoL in both groups at 4 weeks. However, there was 
Table 1 Demographic and Clinical Characteristics

\begin{tabular}{|c|c|c|c|}
\hline Characteristic & Physiotherapy $(n=42)(\%)$ & Yoga therapy $(n=38)(\%)$ & $p$ value \\
\hline Age (years) (mean \pm sd) & $41.4 \pm 12.40$ & $42.6 \pm 11.59$ & 0.65 \\
\hline \multicolumn{4}{|l|}{$\operatorname{Sex}$} \\
\hline Male & $40(95.3)$ & $36(94.7)$ & \multirow[t]{2}{*}{0.94} \\
\hline Female & $2(4.7)$ & $2(5.3)$ & \\
\hline Duration of injury (hours) (mean \pm sd) & $6.5 \pm 7.17$ & $8.0 \pm 7.44$ & 0.15 \\
\hline \multicolumn{4}{|l|}{ Mechanism of injury (MOI) } \\
\hline RTI & $28(66.7)$ & $26(69.2)$ & \multirow[t]{4}{*}{0.72} \\
\hline Fall & $11(26.3)$ & $7(18.0)$ & \\
\hline Assault & $2(4.7)$ & $3(7.5)$ & \\
\hline Fall of heavy object & $1(2.3)$ & $2(5.3)$ & \\
\hline Chest wall tenderness & $42(100)$ & $38(100)$ & - \\
\hline Clinical pneumothorax & $11(26.2)$ & $24(64.0)$ & $<0.001$ \\
\hline Clinical hemothorax & $1(2.4)$ & $4(12.8)$ & 0.07 \\
\hline Surgical emphysema & $9(18.4)$ & $8(22.2)$ & 0.85 \\
\hline Chest tube insertion & $26(61.9)$ & $29(74.4)$ & 0.23 \\
\hline Chest tube duration (median-IQR) (days) & $3(2.8-4)$ & $4(3-5.8)$ & 0.05 \\
\hline Epidural analgesia (n) & 14 & 17 & - \\
\hline \multicolumn{4}{|l|}{ CT rib fracture } \\
\hline No rib fracture & $3(7.1)$ & $2(5.1)$ & \multirow[t]{5}{*}{0.62} \\
\hline $1-3$ rib fracture & $8(19.0)$ & $8(23.0)$ & \\
\hline $3-6$ rib fracture & $22(52.4)$ & $19(48.7)$ & \\
\hline 3 ribs bilateral & 0 & $2(5.1)$ & \\
\hline Flail chest & $9(21.4)$ & $7(17.9)$ & \\
\hline \multicolumn{4}{|l|}{ CT contusion } \\
\hline None & $23(54.8)$ & $22(56.4)$ & \\
\hline 1 lobe, unilateral & $15(35.7)$ & $7(18.1)$ & \\
\hline 1 lobe bilateral/ 2 lobes unilateral & $3(7.1)$ & $6(15.4)$ & 0.21 \\
\hline 2 lobes bilateral & $1(2.4)$ & $2(7.7)$ & \\
\hline 2lobes bilateral & 0 & $1(2.6)$ & \\
\hline \multicolumn{4}{|l|}{ CT pleural involvement } \\
\hline None & $8(19.1)$ & $6(15.4)$ & \\
\hline Pneumothorax & $11(26.2)$ & $9(23.0)$ & \\
\hline Hemo/Hemopneumothorax, unilateral & $19(45.2)$ & $15(38.5)$ & 0.42 \\
\hline Hemo/Hemopneumothorax, bilateral & $4(9.5)$ & $8(23.1)$ & \\
\hline Tension pneumothorax & 0 & 0 & \\
\hline ISS (median-IQR) & $9(9-9)$ & $9(9-9)$ & 0.70 \\
\hline NISS (median-IQR) & $18(9-22)$ & $18(10-22)$ & 0.89 \\
\hline TTSS Score (median-IQR) & $6(5-9)$ & $8(5-10)$ & 0.27 \\
\hline \multicolumn{4}{|l|}{ TTSS Score Grade (n, \%) } \\
\hline Low grade & $34(80.9)$ & $29(74.4)$ & \multirow[t]{2}{*}{0.48} \\
\hline High grade & $8(19.1)$ & $9(25.6)$ & \\
\hline
\end{tabular}

${ }^{*} R T I R o a d$ Traffic Injuries, CT computed tomography, ISS Injury Severity Score, NISS New Injury Severity Score, TTSS Thoracic Trauma Severity Score

significant improvement in the physical domain of QoL at 4 weeks effectively nullifying the negative difference present at baseline in Yogatherapy group. The baseline physical domain of quality of life was statistically less in
$Y T P$ group $(49.4 \pm 16.91)$ as compared to CPT group $(55.10 \pm 10.72)$ with $p=0.05$ at the time of recruitment. However, with time, there was improvement in physical 
Table 2 Respiratory, cytokine and quality of life parameters at admission in both groups

\begin{tabular}{|c|c|c|c|}
\hline Characteristic & Physiotherapy $(n=42)$ & Yoga therapy $(n=38)$ & $p$ value \\
\hline \multicolumn{4}{|l|}{ Pulmonary function tests: } \\
\hline VT (ltrs) & $0.37 \pm 0.12$ & $0.41 \pm 0.2$ & 0.24 \\
\hline FVC & $35.3 \pm 9.10$ & $38.1 \pm 8.90$ & 0.16 \\
\hline FEV1 & $35.8 \pm 10.63$ & $38.7 \pm 8.20$ & 0.16 \\
\hline PEF & $33.9 \pm 9.80$ & $34.4 \pm 8.45$ & 0.82 \\
\hline FEV1/FVC & $106.5 \pm 17.20$ & $107.1 \pm 12.20$ & 0.85 \\
\hline Respiratory muscle endurance: MVV (ltrs) & $25.6 \pm 8.5$ & $27.1 \pm 7.22$ & 0.40 \\
\hline \multicolumn{4}{|l|}{ Chest wall expansion $(\mathrm{cm})$ : } \\
\hline Axillary cirtometry & $3.0 \pm 0.32$ & $2.9 \pm 0.39$ & 0.15 \\
\hline Thoracic cirtometry & $3.8 \pm 0.28$ & $3.7 \pm 0.41$ & 0.14 \\
\hline \multicolumn{4}{|c|}{ Respiratory muscle strength: Diaphragm motion at normal breathing $(\mathrm{mm})$} \\
\hline Right & $15.4 \pm 4.14$ & $15.4 \pm 4.81$ & 0.99 \\
\hline Left & $20.5 \pm 5.89$ & $21.0 \pm 6.55$ & 0.73 \\
\hline \multicolumn{4}{|l|}{ Diaphragm motion at deep breathing $(\mathrm{mm})$} \\
\hline Right & $21.7 \pm 6.15$ & $21.9 \pm 5.53$ & 0.86 \\
\hline Left & $27.3 \pm 6.20$ & $30.1 \pm 9.7$ & 0.12 \\
\hline \multicolumn{4}{|l|}{ Diaphragm thickness at normal breathing ( $\mathrm{mm})$} \\
\hline Right & $1.77 \pm 0.52$ & $1.82 \pm 0.45$ & 0.65 \\
\hline Left & $1.62 \pm 0.44$ & $1.62 \pm 0.39$ & 0.94 \\
\hline \multicolumn{4}{|l|}{ Diaphragm thickness at deep breathing $(\mathrm{mm})$} \\
\hline Right & $2.08 \pm 0.6$ & $2.1 \pm 0.56$ & 0.88 \\
\hline Left & $1.93 \pm 0.64$ & $1.98 \pm 0.62$ & 0.69 \\
\hline \multicolumn{4}{|l|}{ Cytokines } \\
\hline IL-2 & $633.0 \pm 1360.98$ & $670.5 \pm 1209.8$ & 0.48 \\
\hline IL-4 & $1065.8 \pm 5670.7$ & $315.8 \pm 351.20$ & 0.41 \\
\hline IL-8 & $673.5 \pm 1295.10$ & $1833.2 \pm 4317.73$ & 0.12 \\
\hline IL-10 & $100.91 \pm 112.88$ & $99.2 \pm 103.78$ & 0.93 \\
\hline IL-12 & $501.4 \pm 814.36$ & $360.3 \pm 659.56$ & 0.73 \\
\hline TNF- $\alpha$ & $201.3 \pm 433.04$ & $300.4 \pm 589.90$ & 0.54 \\
\hline IFN- $\gamma$ & $288.9 \pm 396.27$ & $323.8 \pm 432.43$ & 0.60 \\
\hline \multicolumn{4}{|l|}{ WHO QoL: } \\
\hline Physical & $55.6 \pm 10.72$ & $49.4 \pm 16.91$ & 0.05 \\
\hline Psychological & $64.3 \pm 13.57$ & $61.9 \pm 17.65$ & 0.51 \\
\hline Social relations & $60.2 \pm 14.67$ & $60.8 \pm 20.96$ & 0.88 \\
\hline Environmental & $51.0 \pm 13.80$ & $51.1 \pm 15.70$ & 0.98 \\
\hline
\end{tabular}

${ }^{*} V T$ Tidal Volume, $F V C$ Forced Vital Capacity, FEV1 Forced Expiratory Volume in one Second, PEF Peak Expiratory Force, $M V V$ Maximum voluntary ventilation, $I L$ Interleukin, $T N F-\alpha$ Tumor Necrosis Factor-a, IFN- $\gamma$ Interferon- $\gamma$, WHO QoL World Health Organization Quality of Life

domain among YTP cohort and was comparable to physiotherapy group $(p=0.13)$.

There was significant improvement of the RME (MVV) $(p=0.003)$ and axillary component of CWM $(p=0.009)$. There was only a relative improvement in RMS as evidenced by the improvement in right diaphragm motion at resting respiration $(p=0.06)$ and during forced respiration $(p=0.06)$. The cytokine levels didn't show any difference in both groups. (Table 4).
Using GEE longitudinal analysis was performed for outcomes to examine the effect of interventions over the time from admission to discharge. (Table 5) There was constant and significant improvement of the FVC [diff. (95\% CI) 4.9 (1.270, 8.09); $p=0.008]$, FEV1 [5.12 (1.256, 8.984); $p=0.009]$, PEF [4.47 $(0.834,8.106) ; p=0.016$ ], $\operatorname{RME}[6.725(3.320,10.245) ; p=<0.0001]$ in the yoga group from discharge to 4 weeks. The change in VT over the time was only marginal $[0.05 \quad(-0.001,0.118)$; $p=0.056]$. 
Table 3 Pulmonary function tests at 4 weeks from discharge

\begin{tabular}{lllcc}
\hline Pulmonary functions & & \\
\hline & Characteristic & Physiotherapy Group & $N=32$ & \multicolumn{1}{l}{ Yogatherapy Group } \\
& & $N=27$ & \\
\hline 1 & VT (Ltrs) & $0.54 \pm 0.15$ & $0.65 \pm 0.24$ & $\mathbf{0 . 0 4}$ \\
2 & FVC $\%$ & $51.6 \pm 11.68$ & $58.5 \pm 11.68$ & $\mathbf{0 . 0 2}$ \\
3 & FEV1\% & $52.5 \pm 13.7$ & $61.6 \pm 13.48$ & $\mathbf{0 . 0 1}$ \\
4 & PEF \% & $42.5 \pm 11.49$ & $53.1 \pm 14.17$ & $\mathbf{0 . 0 0 2}$ \\
5 & FVC/FEV1\% & $106.3 \pm 14.26$ & $109.7 \pm 10.26$ & 0.30 \\
\hline
\end{tabular}

*Independent $t$ test was used, VT Tidal Volume, FVC Forced Vital Capacity, FEV1 Forced Expiratory Volume in one Second, PEF Peak Expiratory Force

Table 4 Quality of Life, Respiratory muscle endurance, Respiratory muscle strength, Chest wall mobility and Cytokine levels at 4 weeks from discharge

\begin{tabular}{|c|c|c|c|c|}
\hline & Characteristic & Physiotherapy Group $N=32$ & Yogatherapy Group $N=27$ & $p$ value \\
\hline \multicolumn{5}{|c|}{ Quality of life } \\
\hline 1 & Physical & $69.7 \pm 14.08$ & $74.73 \pm 9.99$ & 0.13 \\
\hline 2 & Psychological & $76.2 \pm 11.60$ & $79.8 \pm 12.90$ & 0.26 \\
\hline 3 & Social & $65.6 \pm 17.67$ & $70.4 \pm 14.68$ & 0.27 \\
\hline 4 & Environmental & $60.1 \pm 14.97$ & $64.7 \pm 11.68$ & 0.20 \\
\hline \multicolumn{5}{|c|}{ Respiratory muscle endurance } \\
\hline 1 & MVV & $45.9 \pm 13.44$ & $56.4 \pm 12.43$ & 0.003 \\
\hline \multicolumn{5}{|c|}{ Respiratory muscle strength (in millimeters) } \\
\hline 1 & Diaphragm motion at normal breathing (Right) & $18.85 \pm 5.02$ & $21.15 \pm 4.05$ & 0.06 \\
\hline 2 & Diaphragm motion at normal breathing (Left) & $25.50 \pm 7.44$ & $26.14 \pm 4.67$ & 0.70 \\
\hline 3 & Diaphragm motion at deep breathing (Right) & $25.90 \pm 7.08$ & $29.03 \pm 5.16$ & 0.06 \\
\hline 4 & Diaphragm motion at deep breathing (Left) & $32.22 \pm 7.55$ & $35.47 \pm 6.57$ & 0.08 \\
\hline 5 & Diaphragm thickness at normal breathing (Right) & $1.95 \pm 0.57$ & $2.04 \pm 0.32$ & 0.48 \\
\hline 6 & Diaphragm thickness at normal breathing (Left) & $1.79 \pm 0.47$ & $1.83 \pm 0.35$ & 0.68 \\
\hline \multicolumn{5}{|c|}{ Chest Wall mobility (in Centimeters) } \\
\hline 1 & Axillary Cirtometry & $3.6 \pm 0.4$ & $3.9 \pm 0.35$ & 0.009 \\
\hline 2 & Thoracic Cirtometry & $4.5 \pm 0.41$ & $4.7 \pm 0.41$ & 0.057 \\
\hline \multicolumn{5}{|c|}{ Cytokine levels } \\
\hline 1 & IL2 & $317.4 \pm 660.15$ & $556.6 \pm 1090.57$ & 0.13 \\
\hline 2 & IL4 & $1202.8 \pm 5770.68$ & $1389.6 \pm 5650.02$ & 0.97 \\
\hline 3 & IL8 & $665.8 \pm 1482.93$ & $2948.4 \pm 11,688.71$ & 0.85 \\
\hline 4 & IL10 & $108.5 \pm 92.93$ & $80.7 \pm 70.31$ & 0.23 \\
\hline 5 & IL12 & $738.5 \pm 2447.03$ & $1138.8 \pm 3972.88$ & 0.67 \\
\hline 6 & TNF- $\alpha$ & $89.9 \pm 73.79$ & $248.6 \pm 511.81$ & 0.19 \\
\hline 7 & $\mathrm{IFN}-\gamma$ & $242.7 \pm 314.84$ & $355.2 \pm 520.36$ & 0.33 \\
\hline
\end{tabular}

*Independent $\mathrm{t}$ test was used, $M V V$ Maximum voluntary ventilation, $I L$ Interleukin, $T N F-\alpha$ Tumor Necrosis Factor-a, $I F N-\gamma$ Interferon- $\gamma$,

Analysis of cytokines trends during hospital stay before and after interventions in both groups showed inconsistent changes in trends with no significant differences except for IL-4 on day 1 , IL-10 on day 3 which could be a chance finding. During follow up at 4 weeks levels of cytokines were comparable between both the groups. (Table 6). 
Table 5 Change in pulmonary functions and respiratory muscle endurance, chest wall mobility over time from admission to 4 weeks follow-up

\begin{tabular}{|c|c|c|c|c|c|c|}
\hline Characteristic & Group & $\begin{array}{l}\text { At admission* } \\
N=42, N=38\end{array}$ & $\begin{array}{l}\text { Day of Discharge* } \\
N=42, N=38\end{array}$ & $\begin{array}{l}4 \text { Weeks* } \\
N=32, N=27\end{array}$ & Diff. (95\% CI) $¥$ & $\mathrm{p}$ value \\
\hline \multirow[t]{3}{*}{ VT (Ltrs) } & Physio & $0.37 \pm 0.12$ & $0.47 \pm 0.17$ & $0.54 \pm 0.15$ & $0.05(-0.001,0.118)$ & 0.056 \\
\hline & Yoga & $0.41 \pm 0.2$ & $0.51 \pm 0.16$ & $0.65 \pm 0.24$ & & \\
\hline & $p$ value & 0.25 & 0.34 & 0.04 & & \\
\hline \multirow[t]{3}{*}{ FVC } & Physio & $35.3 \pm 9.10$ & $41.9 \pm 10.96$ & $51.6 \pm 11.68$ & $4.9(1.270,8.609)$ & 0.008 \\
\hline & Yoga & $38.1 \pm 8.90$ & $46.4 \pm 10.41$ & $58.5 \pm 11.68$ & & \\
\hline & $p$ value & 0.16 & 0.06 & 0.02 & & \\
\hline \multirow[t]{3}{*}{ FEV1 } & Physio & $35.8 \pm 10.63$ & $41.9 \pm 10.84$ & $52.5 \pm 13.7$ & $5.12(1.256,8.984)$ & 0.009 \\
\hline & Yoga & $38.7 \pm 8.20$ & $45.2 \pm 10.64$ & $61.6 \pm 13.48$ & & \\
\hline & $p$ value & 0.16 & 0.18 & 0.01 & & \\
\hline \multirow[t]{3}{*}{ PEF } & Physio & $33.9 \pm 9.80$ & $37.8 \pm 10.35$ & $42.5 \pm 11.49$ & $4.47(0.834,8.106)$ & 0.016 \\
\hline & Yoga & $34.4 \pm 8.45$ & $41.8 \pm 10.76$ & $53.1 \pm 14.17$ & & \\
\hline & $p$ value & 0.82 & 0.09 & 0.002 & & \\
\hline \multirow[t]{3}{*}{ FVC/FEV1 } & Physio & $106.5 \pm 17.20$ & $105.0 \pm 12.96$ & $106.3 \pm 14.26$ & $1.003(-3.069,5.076)$ & 0.629 \\
\hline & Yoga & $107.1 \pm 12.20$ & $104.7 \pm 12.67$ & $109.7 \pm 10.26$ & & \\
\hline & $p$ value & 0.85 & 0.93 & 0.30 & & \\
\hline \multirow[t]{3}{*}{ MVV } & Physio & $25.6 \pm 8.50$ & $31.9 \pm 8.82$ & $45.9 \pm 13.44$ & $6.725(3.320,10.245)$ & $<\mathbf{0 . 0 0 0 1}$ \\
\hline & Yoga & $27.1 \pm 7.22$ & $40.5 \pm 10.27$ & $56.4 \pm 12.43$ & & \\
\hline & $p$ value & 0.40 & 0.0001 & 0.003 & & \\
\hline \multirow[t]{3}{*}{ Axillary Cirtometry (cm) } & Physio & $3.0 \pm 0.32$ & $3.3 \pm 0.39$ & $3.6 \pm 0.4$ & $0.071(-0.071,0.214)$ & 0.33 \\
\hline & Yoga & $2.9 \pm 0.39$ & $3.4 \pm 0.33$ & $3.9 \pm 0.35$ & & \\
\hline & $p$ value & 0.15 & 0.10 & 0.009 & & \\
\hline \multirow[t]{3}{*}{ Thoracic Cirtometry $(\mathrm{cm})$} & Physio & $3.8 \pm 0.28$ & $4.1 \pm 0.35$ & $4.5 \pm 0.41$ & $0.056(-0.095,0.208)$ & 0.46 \\
\hline & Yoga & $3.7 \pm 0.41$ & $4.2 \pm 0.42$ & $4.7 \pm 0.41$ & & \\
\hline & $p$ value & 0.14 & 0.19 & 0.057 & & \\
\hline
\end{tabular}

*Independent $\mathrm{t}$ test; ¥ Generalized estimation equation analysis, VT Tidal Volume, FVC Forced Vital Capacity, FEV1 Forced Expiratory Volume in one Second, $P E F$ Peak Expiratory Force, $M V V$ Maximum voluntary ventilation

\section{Discussion}

Prior to conceptualizing this study design, extensive literature review did not yield any studies pertaining to effects of yoga in trauma patients. However, there were studies involving yoga and other mind body techniques (MBT) evaluating the beneficial effects on pulmonary functions, RME, RMS, QoL and immune markers in non-trauma patients.

In our study, demographic profiles in both groups were comparable. The mean length of hospital stay (LoS) was 3.7 days in CPT group and 4.3 days in YTP group. AlKoudmani et al. reported 4.5 days of mean LoS in chest injury patients [5]. However, Demirhan R et al. and Narayanan $\mathrm{R}$ et al. reported mean hospital stay of 10.7 days and 10.09 days, respectively [6, 52]. But, Park HB et al. and Bagaria et al. reported longer and varying LoS 13.5-27 days and 20.4-29 days, respectively [14, 53]. These differences could be because of associated multisystem injuries. In our study, shorter hospital stay could be because of recruitment of isolated blunt chest injury patients. YTP group had longer duration of LoS compared to CPT group though nonsignificant statistically. This could primarily be due to the need of chest tube for longer duration in YTP group compared to CPT group which was statistically significant. Secondly, patients in YTP group had significantly higher percentage of clinically detected pneumothorax and hemothorax requiring chest tube drainage in the primary survey. These could be wellcorroborated signifying the severity. However, this difference was by chance because of random allocation of the patients in two groups.

Chest trauma impairs pulmonary dynamics and further negatively affects pulmonary functions [54, 55]. Hence, early chest physiotherapy effectively modulates pulmonary dynamics and hence the pulmonary functions, respiratory strength and endurance. 
Table 6 Changes in Cytokines levels before and after intervention during hospital stay (in $\mathrm{pg} / \mathrm{ml}$

\begin{tabular}{|c|c|c|c|c|c|c|c|c|c|c|}
\hline \multirow[t]{2}{*}{ Characteristic } & \multirow[t]{2}{*}{ Group } & \multicolumn{3}{|l|}{ Day 1} & \multicolumn{3}{|l|}{ Day 2} & \multicolumn{3}{|l|}{ Day 3} \\
\hline & & $\begin{array}{l}\text { Before } \\
N=42,38\end{array}$ & $\begin{array}{l}\text { After } \\
N=42,38\end{array}$ & $p$ value & $\begin{array}{l}\text { Before } \\
N=31,32\end{array}$ & $\begin{array}{l}\text { After } \\
N=29,32\end{array}$ & $p$ value & $\begin{array}{l}\text { Before } \\
N=10,17\end{array}$ & $\begin{array}{l}\text { After } \\
N=9,14\end{array}$ & $P$ value \\
\hline \multirow[t]{3}{*}{ IL-2 } & Physio & $\begin{array}{l}753.3 \pm \\
1423.95\end{array}$ & $\begin{array}{l}651.6 \pm \\
1373.15\end{array}$ & 0.53 & $\begin{array}{l}723.5 \pm \\
1906.82\end{array}$ & $\begin{array}{l}513.1 \pm \\
1015.55\end{array}$ & 0.34 & $\begin{array}{l}1216.2 \\
\quad \pm 1718.47\end{array}$ & $\begin{array}{r}1096.0 \pm \\
1752.45\end{array}$ & 0.36 \\
\hline & Yoga & $\begin{array}{l}565.9 \pm \\
1049.54\end{array}$ & $\begin{array}{l}710.4 \pm \\
1198.87\end{array}$ & 0.29 & $\begin{array}{r}1143.3 \pm \\
1738.4\end{array}$ & $\begin{array}{l}821.0 \pm \\
1657.77\end{array}$ & 0.14 & $\begin{array}{r}693.5 \pm \\
881.49\end{array}$ & $\begin{array}{r}1327.8 \pm \\
2380.37\end{array}$ & 0.26 \\
\hline & $\mathrm{p}$ & 0.95 & 0.80 & - & 0.36 & 0.32 & - & 0.72 & 0.80 & - \\
\hline \multirow[t]{3}{*}{ IL-4 } & Physio & $\begin{array}{r}292.3 \pm \\
454.33\end{array}$ & $\begin{array}{r}184.3 \pm \\
309.19\end{array}$ & 0.05 & $\begin{array}{r}266.8 \pm \\
768.48\end{array}$ & $\begin{array}{r}1291.6 \pm \\
6139.26\end{array}$ & 0.32 & $\begin{array}{l}399.2 \pm \\
366.66\end{array}$ & $\begin{array}{r}609.9 \pm \\
708.91\end{array}$ & 0.31 \\
\hline & Yoga & $\begin{array}{r}1035.6 \pm \\
4719.21\end{array}$ & $\begin{array}{r}418.0 \pm \\
557.52\end{array}$ & 0.42 & $\begin{array}{r}1265.1 \pm \\
5121.5\end{array}$ & $\begin{array}{l}1157.3 \\
\quad \pm 4753.25\end{array}$ & 0.93 & $\begin{array}{l}608.0 \pm \\
1273.26\end{array}$ & $\begin{array}{r}2573.8 \pm \\
8194.09\end{array}$ & 0.33 \\
\hline & $p$ & 0.31 & 0.02 & - & 0.29 & 0.92 & - & 0.61 & 0.70 & - \\
\hline \multirow[t]{3}{*}{ IL-8 } & Physio & $\begin{array}{l}691.9 \pm \\
1314.02\end{array}$ & $\begin{array}{r}1211.0 \pm \\
2352.53\end{array}$ & 0.15 & $\begin{array}{l}858.7 \pm \\
1920.63\end{array}$ & $\begin{array}{r}418.4 \pm \\
878.51\end{array}$ & 0.21 & $\begin{array}{r}338.4 \pm \\
320.33\end{array}$ & $\begin{array}{r}343.7 \pm \\
275.45\end{array}$ & 0.67 \\
\hline & Yoga & $\begin{array}{l}7408.5 \pm \\
39582.86\end{array}$ & $\begin{array}{l}8023.5 \pm \\
\quad 42902.06\end{array}$ & 0.27 & $\begin{array}{r}1638.3 \pm \\
3033.43\end{array}$ & $\begin{array}{r}2684.4 \pm \\
6610.75\end{array}$ & 0.32 & $\begin{array}{l}747.7 \pm \\
1478.88\end{array}$ & $\begin{array}{l}705.4 \pm \\
1500.78\end{array}$ & 0.39 \\
\hline & $p$ & 0.40 & 0.38 & - & 0.23 & 0.06 & - & 0.61 & 0.97 & - \\
\hline \multirow[t]{3}{*}{ IL-10 } & Physio & $111.3 \pm 126$ & $\begin{array}{r}103.1 \pm \\
145.81\end{array}$ & 0.68 & $\begin{array}{l}91.6 \pm \\
133.79\end{array}$ & $\begin{array}{c}82.8 \pm \\
93.48\end{array}$ & 0.63 & $\begin{array}{r}85.7 \pm \\
74.81\end{array}$ & $\begin{array}{r}68.0 \pm \\
62.44\end{array}$ & 0.12 \\
\hline & Yoga & $\begin{array}{r}116.2 \pm \\
118.12\end{array}$ & $\begin{array}{r}130.3 \pm \\
135.24\end{array}$ & 0.33 & $\begin{array}{r}121.2 \pm \\
109.16\end{array}$ & $\begin{array}{r}94.6 \pm \\
88.22\end{array}$ & 0.11 & $\begin{array}{r}87.2 \pm \\
72.83\end{array}$ & $\begin{array}{r}116.9 \pm \\
106.35\end{array}$ & 0.07 \\
\hline & $\mathrm{P}$ & 0.86 & 0.40 & - & 0.10 & 0.61 & - & 0.96 & 0.23 & - \\
\hline \multirow[t]{3}{*}{ IL-12 } & Physio & $\begin{array}{l}776.9 \pm \\
1872.91\end{array}$ & $\begin{array}{r}1235.7 \pm \\
4119.79\end{array}$ & 0.28 & $\begin{array}{l}886.3 \pm \\
3542.33\end{array}$ & $\begin{array}{l}917.0 \pm \\
3268.52\end{array}$ & 0.98 & $\begin{array}{l}767.9 \pm \\
1196.07\end{array}$ & $\begin{array}{r}462.6 \pm \\
788.79\end{array}$ & 0.93 \\
\hline & Yoga & $\begin{array}{r}278.7 \pm \\
509.22\end{array}$ & $\begin{array}{r}339.7 \pm \\
524.03\end{array}$ & 0.60 & $\begin{array}{l}1589.1 \\
\quad \pm 3983.47\end{array}$ & $\begin{array}{l}1666.8 \\
\quad \pm 4741.33\end{array}$ & 0.93 & $\begin{array}{l}843.6 \pm \\
1216.19\end{array}$ & $\begin{array}{r}1393.3 \pm \\
2747.07\end{array}$ & 0.53 \\
\hline & $p$ & 0.12 & 0.19 & - & 0.46 & 0.48 & - & 0.58 & 0.34 & - \\
\hline \multirow[t]{3}{*}{ TNF- $\alpha$} & Physio & $\begin{array}{r}360.3 \pm \\
879.62\end{array}$ & $\begin{array}{l}470.2 \pm \\
1270.06\end{array}$ & 0.66 & $\begin{array}{r}252.4 \pm \\
577.88\end{array}$ & $\begin{array}{r}142.4 \pm \\
143.34\end{array}$ & 0.25 & $\begin{array}{r}505.8 \pm \\
898.25\end{array}$ & $\begin{array}{r}314.9 \pm \\
506.89\end{array}$ & 0.55 \\
\hline & Yoga & $\begin{array}{l}279.8 \pm \\
\quad 750.24\end{array}$ & $\begin{array}{r}246.7 \pm \\
445.58\end{array}$ & 0.81 & $\begin{array}{r}389.6 \pm \\
827.86\end{array}$ & $\begin{array}{l}464.4 \pm \\
1042.97\end{array}$ & 0.74 & $\begin{array}{r}355.2 \pm \\
596.82\end{array}$ & $\begin{array}{l}278.0 \pm \\
363.85\end{array}$ & 0.51 \\
\hline & $p$ & 0.95 & 0.29 & - & 0.45 & 0.09 & - & 0.60 & 0.84 & - \\
\hline \multirow[t]{3}{*}{ IFN- $\gamma$} & Physio & $\begin{array}{r}305.7 \pm \\
441.85\end{array}$ & $\begin{array}{r}329.3 \pm \\
410.17\end{array}$ & 0.81 & $\begin{array}{r}288.2 \pm \\
381.56\end{array}$ & $\begin{array}{r}185.1 \pm \\
280.6\end{array}$ & 0.43 & $\begin{array}{r}455.8 \pm \\
551.25\end{array}$ & $\begin{array}{r}\mathrm{b} 324.4 \pm \\
340.52\end{array}$ & 0.46 \\
\hline & Yoga & $\begin{array}{r}286.4 \pm \\
378.29\end{array}$ & $\begin{array}{r}352.3 \pm \\
420.59\end{array}$ & 0.30 & $350.5 \pm 336$ & $\begin{array}{r}392.4 \pm \\
446.02\end{array}$ & 0.58 & $\begin{array}{r}409.2 \pm \\
497.35\end{array}$ & $\begin{array}{r}254.9 \pm \\
264.43\end{array}$ & 0.42 \\
\hline & $p$ & 0.90 & 0.97 & - & 0.50 & 0.03 & - & 0.82 & 0.60 & - \\
\hline
\end{tabular}

*Independent $t$ test; ¥ Generalized estimation equation analysis, $I L$ Interleukin, $T N F-\alpha$ Tumor Necrosis Factor-a, $I F N-\gamma$ Interferon- $\gamma$

In multiple studies involving non-trauma patients, Pranayama have shown improvement in pulmonary functions, RMS and RME. Joshi LN et al. showed significant improvement in FVC, PEF rate and MVV with 6 weeks of Pranayama in healthy volunteers [33]. Another study also documented significant improvement in FVC, FEV1, PEF and MVV with 60 days of Pranayama [34]. Another randomized study in asthmatics showed significant improvements in FVC, FEV1 and PEF with 6 months of Yogatherapy [35]. In our study, there was significant improvement in pulmonary functions at 4 weeks in $\mathrm{Yo}$ gatherapy group.

Several inspiratory muscle training programs (IMT) have been used to improve the strength and endurance of chest wall muscles in elderly; and in patients with chronic obstructive pulmonary disease (COPD), neuromuscular diseases and stroke. Cebriài Iranzo Md et al. found significant improvement in MVV in elderly patients with Pranayama [37]. Beckerman $\mathrm{M}$ et al. demonstrated significant improvement in exercise capacity and QoL using IMT in COPD patients and decrease in dyspnea, primary 
health-care use and hospitalization days [56]. Bissett BM et al. and Sutbeyaz ST et al. demonstrated greater improvements in inspiratory strength using IMT in mechanically ventilated and stroke patients, respectively $[57,58]$. Yi SJ et al. have demonstrated sling aerobic exercises as an alternative for RMS training [59]. Jung JH et al. showed significant correlation between diaphragm thickness on ultrasound, diaphragmatic excursion, and pulmonary functions in patients with chronic stroke [60].

In our study, there was significant improvement in RME in YTP group; and it was evident at early stage and persisted throughout the study. There was only marginal improvement in RMS in YTP group at 4 weeks as evidenced by improvement in right diaphragm motion both at resting and deep respiration. This further indicates that if Yogatherapy continued for longer duration could potentially improve the strength of respiratory muscles.

CWM is an indirect measure of thoracic wall compliance and reflects the lung functions [61]. There was significant improvement in chest wall mobility in patients receiving Yoga therapy in our study.

The literature is abundant with various cytokines and their significance in immediate post-injury catastrophic events, and long-term outcomes [62]. Different MBTs including yoga have been shown to affect cytokines activity at circulating, cellular and genomic level [31]. Circulating cytokines variations are transient phenomenon and levels vary even in small scale insults to body. For changes to be apparent clinically, these MBT interventions need to be carried for longer duration. In our study, circulating cytokines were assessed and found no significant difference in cytokine trends between both groups. The follow-up was very short to show difference in cytokine profiles between the groups. Thus, for changes in circulating cytokines to be reflected, longer duration of followup or genomic markers' expressions need to be studied.

Post-traumatic health-related quality of life is significant problem and is all-time low in trauma patients. There is no single standard unified QoL questionnaire particular to trauma and many QoL questionnaires such as WHO QoL BREF, SF-12, Euro-QoL, custom designed have been used but none have predictive ability for long-term outcomes [63]. In our study, we found there was significant improvement in physical domain of QoL in Yoga group. But this needs to be followed up for longer duration to see the actual effect. It is worth noting that it is not possible to assess patients QoL before the traumatic events. With the available QoL questionnaires, there is always bias of present traumatic event confounding actual QoL.

This single center study showed that addition of Yogatherapy to standard chest physiotherapy significantly improves pulmonary functions (FVC, FEV and PEF) at early stage of hospitalization. Yogatherapy also improves post injury QoL, RMS, RME, and CWM significantly as compared to chest physiotherapy alone. However, Yogatherapy had no impact on circulating cytokines. This study gives insights for sustained and positive long-term outcomes using Yogatherapy as additional rehabilitation strategy in injured patients.

\section{Declarations}

Conflict of interest The authors declare that they have no conflict of interest.

Ethical approval Ethics approval was taken from the Institutional Ethics Committee.

Informed consent Informed consent was obtained from all individual participants included in the study.

Trial registration number Ctri.nic.in/clinicaltrials/login.php, numberREF/2016/05/011287.

Grant support Department of Science and Technology, Government of India.

\section{References}

1. World Health Organization. Violence, Injuries, and Disability: Biennial 2006-2007 Report. Geneva, Switzerland: World Health Organization; 2008. Cited on 2016 May 30. Available fromhttp:// apps.who.int/iris/bitstream/10665/43955/1/9789241597081eng. pdf. Accessed May 30, 2016

2. World Health Organization. Global Status Report on Road Safety 2015. Geneva, Switzerland: World Health Organization; 2015. Cited on 2016 May 30. Available at: http://www.who.int/vio lence_injury_prevention/road_safety_status/2015/GSRRS2015_ Summary_EN_final.pdf. Accessed May 30, 2016

3. World Health Organization. Injuries and Violence: The Facts 2014. Geneva, Switzerland: World Health Organization; 2014. Cited on 2016 May 30. Available at: http://apps.who.int/iris/bit stream/10665/44288/ 1/9789241599375eng.pdf. Accessed May 30,2016

4. Chandran A, Hyder AA, Peek-Asa C (2010) The global burden of unintentional injuries and an agenda for progress. Epidemiol Rev $32: 110-120$

5. Al-Koudmani I, Darwish B, Al-Kateb K et al (2012) Chest trauma experience over eleven-year period at al mouassat university teaching hospital- Damascus: a retrospective review of 888 cases. J Cardiothorac Surg 7:35

6. Demirhan R, Onan B, Oz K et al (2009) Comprehensive analysis of 4205 patients with chest trauma: a 10-year experience. Interact Cardiovasc Thorac Surg 9:450-453

7. O'Connor JV, Adamski J (2010) The diagnosis and treatment of non-cardiac thoracic trauma. J R Army Med Corps 156:5-14

8. Shah JV, Solanki MI (2015) Analytic study of chest injury. IJSS J Surg 1:5-9

9. Shorr RM, Crittenden M, Indeck M et al (1987) Blunt thoracic trauma. analysis of 515 patients. Ann Surg 206:200-205

10. Mohta M, Kumar P, Mohta A et al (2006) Experiences with chest trauma: where do we stand today. Indian J Crit Care Med $10: 25-28$ 
11. Cross LJ, Matthay MA (2011) Biomarkers in acute lung injury: insights into the pathogenesis of acute lung injury. Crit Care Clin 27:355-377

12. Orfanos SE, Mavrommati I, Korovesi I et al (2004) Pulmonary endothelium in acute lung injury: from basic science to the critically ill. Intensive Care Med 30:1702-1714

13. Raghavendran K, Notter RH, Davidson BA et al (2009) Lung contusion: inflammatory mechanisms and interaction with other injuries. Shock 32:122-130

14. Bagaria V, Mathur P, Madan K, et al (2020) Predicting Outcomes After Blunt Chest Trauma-Utility of Thoracic Trauma Severity Score, Cytokines (IL-1 $\beta$, IL-6, IL-8, IL-10, and TNF- $\alpha$ ), and Biomarkers (vWF and CC-16). Indian J Surg (online ahead of print) https://doi.org/https://doi.org/10.1007/s12262-020-024074.

15. Bergeron E, Lavoie A, Clas D et al (2003) Elderly trauma patients with rib fractures are at greater risk of death and pneumonia. J Trauma 54:478-485

16. Pape HC, Remmers D, Rice J et al (2000) Appraisal of early evaluation of blunt chest trauma: development of a standardized scoring system for initial clinical decision making. J Trauma 49:496-504

17. Martínez Casas I, Amador Marchante MA, Paduraru M et al (2016) Thorax trauma severity score: is it reliable for patient's evaluation in a secondary level hospital? Bull Emerg Trauma 4:150-155

18. Bayer J, Lefering R, Reinhardt S et al (2017) Thoracic trauma severity contributes to differences in intensive care therapy and mortality of severely injured patients: analysis based on the trauma register DGU®. World J Emerg Surg 12:43

19. Zahran MR, Elwahab AAEMA, El Nasr MMA et al (2020) Evaluation of the predictive value of thorax trauma severity score (TTSS) in thoracic-traumatized patients. CardiothoracSurg 28:3

20. Subhani SS, Muzaffar MS, Khan MI (2014) Comparison of outcome between low and high thoracic trauma severity score in blunt trauma chest patients. J Ayub Med Coll Abbottabad 26:474-477

21. de Munter L, Polinder S, Haagsma JA et al (2020) Prevalence and prognostic factors for psychological distress after trauma. Arch Phys Med Rehabil 101:877-884

22. Kendrick D, Baker R, Hill T et al (2018) Early risk factors for depression, anxiety and post-traumatic distress after hospital admission for unintentional injury: multicentre cohort study. J Psychosom Res 112:15-24

23. Baecher K, Kangas M, Taylor A et al (2018) The role of site and severity of injury as predictors of mental health outcomes following traumatic injury. Stress Health 34:545-551

24. Baker E, Xyrichis A, Norton C et al (2018) The long-term outcomes and health-related quality of life of patients following blunt thoracic injury: a narrative literature review. Scand J Trauma Resusc Emerg Med 26:67

25. Leone M, Brégeon F, Antonini F et al (2008) Long-term outcome in chest trauma. Anesthesiology 109:864-871

26. Marasco S, Lee G, Summerhayes R et al (2015) Quality of life after major trauma with multiple rib fractures. Injury 46:61-65

27. Sutton PP, Parker RA, Webber BA et al (1983) Assessment of the forced expiration technique, postural drainage and directed coughing in chest physiotherapy. Eur J Respir Dis 64:62-68

28. Castro AA, Calil SR, Freitas SA et al (2013) Chest physiotherapy effectiveness to reduce hospitalization and mechanical ventilation length of stay, pulmonary infection rate and mortality in ICU patients. Respir Med 107:68-74

29. Stiller K (2013) Physiotherapy in intensive care: an updated systematic review. Chest 144:825-847
30. Cramer H, Ward L, Saper R et al (2015) The safety of yoga: a systematic review and meta-analysis of randomized controlled trials. Am J Epidemiol 182:281-293

31. Bower JE, Irwin MR (2016) Mind-body therapies and control of inflammatory biology: a descriptive review. Brain Behav Immun $51: 1-11$

32. Kiecolt-Glaser JK, Christian L, Preston H et al (2010) Stress, inflammation, and yoga practice. Psychosom Med 72:113-121

33. Joshi LN, Joshi VD, Gokhale LV (1992) Effect of short term "Pranayam" practice on breathing rate and ventilatory functions of lung. Indian J Physiol Pharmacol 36:105-108

34. Ahmed QR, Sau SK, Kar SK (2010) An evaluation of pulmonary parameters in two groups of subjects during Yoga practice. Nepal Med Coll J 12:180-182

35. Agnihotri S, Kant S, Kumar S et al (2016) The assessment of effects of yoga on pulmonary functions in asthmatic patients: a randomized controlled study. J Med Soc 30:98-102

36. D'souza C, Avadhany ST (2014) Effects of yoga training and detraining on physical performance measures in prepubertal children: a randomized trial. Indian $\mathrm{J}$ Physiol Pharmacol 58:61-68

37. Cebriài i Iranzo Md, Arnall DA, Igual Camacho C et al (2014) Effects of inspiratory muscle training and yoga breathing exercises on respiratory muscle function in institutionalized frail older adults: a randomized controlled trial. J Geriatr Phys Ther 37:65-75

38. Tran MD, Holly RG, Lashbrook J et al (2001) Effects of hatha yoga practice on the health-related aspects of physical fitness. Prev Cardiol 4:165-170

39. Bulavin VV, Kliuzhev VM, Kliachkin LM, et al (1993) Elements of yoga therapy in the combined rehabilitation of myocardial infarct patients in the functional recovery period. VoprKurortolFizioter Lech FizKult 4:79. (Article in Russian).

40. Vijayaraghava A, Doreswamy V, Narasipur OS et al (2015) Effect of yoga practice on levels of inflammatory markers after moderate and strenuous exercise. J Clin Diagn Res 9:08-12

41. Pullen PR, Nagamia SH, Mehta PK et al (2008) Effects of yoga on inflammation and exercise capacity in patients with chronic heart failure. J Card Fail 14:407-413

42. Morgan N, Irwin MR, Chung M et al (2014) The effects of mindbody therapies on the immune system: meta-analysis. PLoS One 9:100903

43. Seppälä EM, Nitschke JB, Tudorascu DL et al (2014) Breathingbased meditation decreases posttraumatic stress disorder symptoms in U.S. military veterans: a randomized controlled longitudinal study. J Trauma Stress 27:397-405

44. Brown RP, Gerbarg PL (2005) Sudarshan Kriya yogic breathing in the treatment of stress, anxiety, and depression: part I-neurophysiologic model. J Altern Complement Med 11:189-201

45. Ruppel GL, Paul LE (2012) Pulmonary function testing. Respir Care 57:165-175

46. Miller MR, Crapo R, Hankinson J et al (2005) General considerations for lung function testing. Eur Respir J 26:153-161

47. Miller MR, Hankinson J, Brusasco V et al (2005) Standardisation of spirometry. Eur Respir J 26:319-338

48. Culver BH, Graham BL, Coates A (2017) Recommendations for a standardized pulmonary function report. an official american thoracic society technical statement. Am J Respir Crit Care Med 196:1463-1472

49. World Health Organization. Division of Mental Health. (1996). WHOQOL-BREF: introduction, administration, scoring and generic version of the assessment: field trial version, December 1996. World Health Organization. https://apps.who. int/iris/handle/10665/63529 (used with permission)

50. Cardenas LZ, Santana PV, Caruso P et al (2018) Diaphragmatic ultrasound correlates with inspiratory muscle strength and 
pulmonary function in healthy subjects. Ultrasound Med Biol 44:786-793

51. Debouche S, Pitance L, Robert A et al (2016) Reliability and reproducibility of chest wall expansion measurement in young healthy adults. J Manipulative Physiol Ther 39:443-449

52. Narayanan R, Kumar S, Gupta A et al (2018) An analysis of presentation, pattern and outcome of chest trauma patients at an urban level 1 trauma center. Indian J Surg 80:36-44

53. Park HB, Hyun SY, Kim JJ et al (2017) Prognosis of pulmonary function in patients with multiple rib fractures. J Trauma Injury 30:179-185

54. Carrie C, Stecken L, Scotto M et al (2018) Forced vital capacity assessment for risk stratification of blunt chest trauma patients in emergency settings: a preliminary study. Anaesth Crit Care Pain Med 37:67-71

55. Carver TW, Milia DJ, Somberg C et al (2015) Vital capacity helps predict pulmonary complications after rib fractures. J Trauma Acute Care Surg 79:413-416

56. Beckerman M, Magadle R, Weiner M et al (2005) The effects of 1 year of specific inspiratory muscle training in patients with COPD. Chest 128:3177-3182

57. Bissett BM, Leditschke IA, Neeman T et al (2016) Inspiratory muscle training to enhance recovery from mechanical ventilation: a randomised trial. Thorax 71:812-819
58. Sutbeyaz ST, Koseoglu F, Inan L et al (2010) Respiratory muscle training improves cardiopulmonary function and exercise tolerance in subjects with subacute stroke: a randomized controlled trial. Clin Rehabil 24:240-250

59. Yi SJ, Kim JS (2015) The effects of respiratory muscle strengthening exercise using a sling on the amount of respiration. J Phys Ther Sci 27:2121-2124

60. Jung JH, Kim NS (2017) The correlation between diaphragm thickness, diaphragmatic excursion, and pulmonary function in patients with chronic stroke. J Phys Ther Sci 29:2176-2179

61. LanzaFde C, de Camargo AA, Archija LR et al (2013) Chest wall mobility is related to respiratory muscle strength and lung volumes in healthy subjects. Respir Care 58:2107-2112

62. Lord JM, Midwinter MJ, Chen YF et al (2014) The systemic immune response to trauma: an overview of pathophysiology and treatment. Lancet 384(9952):1455-1465

63. Wanner JP, deRoon-Cassini T, Kodadek L et al (2015) Development of a trauma-specific quality-of-life measurement. J Trauma Acute Care Surg 79:275-281

Publisher's Note Springer Nature remains neutral with regard to jurisdictional claims in published maps and institutional affiliations. 